\title{
Prévoir et gérer la coagulopathie et les manifestations thrombotiques de la COVID-19 sévère
}

\author{
Lucas C. Godoy MD, Ewan C. Goligher MD PhD, Patrick R. Lawler MD MSP, Arthur S. Slutsky MD MSc, \\ Ryan Zarychanski MD MSc
}

Citation : CMAJ 2020 October 5;192:E1156-61. doi : 10.1503/cmaj.201240-f; diffusion hâtive le 17 août 2020

Voir la version anglaise de l'article ici : www.cmaj.ca/lookup/doi/10.1503/cmaj.201240

$\mathbf{L}$ a maladie à coronavirus 2019 (COVID-19), qui résulte d'une infection au coronavirus du syndrome respiratoire aigu sévère 2 (SRAS-CoV-2), s'accompagne d'un vaste éventail de manifestations dont l'intensité varie. Même si la plupart des cas ne requièrent pas d'hospitalisation, environ $10 \%-15 \%$ des patients symptomatiques doivent être hospitalisés, dont près de $20 \%$ aux soins intensifs ${ }^{1}$. La thrombose est une caractéristique clinique prédominante de la COVID-19. En effet, de $5 \%$ à $30 \%$ des patients hospitalisés manifestent un événement thrombotique ${ }^{2-4}$ cliniquement avéré. Selon les données émergentes, une atteinte endothéliale résultant de l'envahissement des cellules par le SRAS-CoV-2 et un dérèglement subséquent de la réponse de l'hôte impliquant les voies de l'inflammation et de la coagulation jouent un rôle crucial dans la progression de la COVID-19 sévère ${ }^{5}$. La thrombose macro- et microvasculaire contribuerait aux défaillances organiques, à l'atteinte multisystémique et au décès ${ }^{6,7}$ et pour l'instant, la façon optimale de prévenir et de traiter la thrombose qui accompagne la COVID-19 nous échappe ${ }^{8}$. Nous passons ici en revue les connaissances recueillies à ce jour sur la coagulopathie associée à la COVID-19 (encadré $n^{\circ} 1$ ), les orientations thérapeutiques actuelles et les questions en suspens.

Encadré ${ }^{\circ} 1$ : Données utilisées pour la présente revue Interrogation non systématique de la littérature du réseau MEDLINE et recherche manuelle pour les articles publiés en ligne dans les revues suivantes: Annals of Internal Medicine, Blood, Canadian Journal of Cardiology, CMAJ, Circulation, European Heart Journal, Journal of the American College of Cardiology, Journal of the American Medical Association, Journal of Thrombose and Hemostasis, The Lancet et New England Journal of Medicine. Nous avons évité de citer des études en prépublication qui n'ont pas encore été révisées par des pairs, à l'exception de 3 articles ${ }^{34,63,64}$.

\section{POINTS CLÉS}

- La maladie à coronavirus 2019 (COVID-19) est associée à une atteinte endothéliale et à l'hypercoagulabilité.

- Les anomalies fréquentes aux analyses de laboratoire pour la coagulopathie liée à la COVID-19 incluent l'augmentation des taux de D-dimères et de fibrinogène et l'allongement du temps de thromboplastine partielle activée.

- Des complications thrombotiques veineuses et artérielles peuvent survenir plus souvent avec la COVID-19 qu'avec d'autres insuffisances respiratoires.

- L'anticoagulothérapie à dose thromboprophylactique est largement recommandée chez les patients atteints de COVID-19; des essais cliniques sont en cours pour vérifier si l'anticoagulothérapie à dose plus élevée peut être bénéfique.

\section{Comment le SRAS-CoV-2 entraîne-t-il la coagulopathie?}

Même si notre compréhension des mécanismes qui sous-tendent la coagulopathie liée à la COVID-19 évolue rapidement, une hypothèse récente évoque un lien en cascade entre lésions endothéliales, activation inflammatoire et immunitaire et coagulation (figure $1 A)^{9,10}$. Le SRAS-CoV-2 pénètre les cellules de l'hôte par le biais d'une interaction entre la protéine $S$ virale et le récepteur de l'enzyme de conversion de l'angiotensine 2 (ECA2) exprimé dans de nombreux organes et tissus, y compris dans les pneumocytes de type II (qui sécrètent le surfactant pulmonaire), le cerveau, le cœur, les reins et l'endothelium ${ }^{11,12}$. L'ECA2 se décompose normalement en angiotensine $\mathrm{II}$, et la régulation à la baisse de l'ECA2 médiée par le SRAS-CoV-2 pourrait entraîner une accumulation d'angiotensine II et contribuer ainsi à un état procoagulant ${ }^{13}$. L'atteinte endothéliale déclenchée par l'entrée du SRASCoV-2 dans ces cellules jouerait un rôle clé et expliquerait probablement les signes anatomopathologiques d'endothélite 
diffuse dans plusieurs organes, dont les poumons, les reins, le cœur et les intestins ${ }^{5}$. L'endothéliopathie peut causer une réponse inflammatoire chez l'hôte, caractérisée par une activation immunitaire excessive et un orage cytokinique propices à l'hypercoagulabilité et à la thrombose ${ }^{14,15}$. L'activation des macrophages, l'expression du facteur tissulaire et la cascade inflammatoire associée (mettant entre autre à contribution la voie de signalisation de l'interleukine 6) sont caractéristiques de l'infection au SRAS-CoV-2 sévère ${ }^{8,10}$.

Étant donné les liens inextricables entre coagulation et inflammation, les $D$-dimères, le fibrinogène et les cytokines inflammatoires sont en corrélation dans la COVID-19 ${ }^{16}$. Le SRASCoV-2 active les voies du complément, ce qui aggrave l'atteinte des cellules endothéliales, l'activation plaquettaire et la throm- bose ${ }^{17}$. Dans des modèles animaux d'infection à coronavirus, l'inhibition des médiateurs de l'inflammation dans la voie du complément, particulièrement les anaphylatoxines $\mathrm{C} 3 \mathrm{a}$ et $\mathrm{C} 5 \mathrm{a}$, pourrait contribuer à réduire les taux de cytokines, la charge virale et l'atteinte pulmonaire ${ }^{18}$. Outre la lésion endothéliale directe, l'activation du complément peut donner lieu à une microangiopathie thrombotique en induisant l'expression du facteur tissulaire, la sécrétion du facteur de Von Willebrand et la sécrétion/activation du facteur $\mathrm{V}^{19,20}$. Les anticorps antiphospholipides et les anticorps anticoagulants lupiques ont aussi été détectés chez certains patients atteints de COVID-19 et pourraient contribuer à la survenue des événements thrombotiques, même si la prévalence et la portée clinique de ces anticorps restent à vérifier ${ }^{21-23}$.

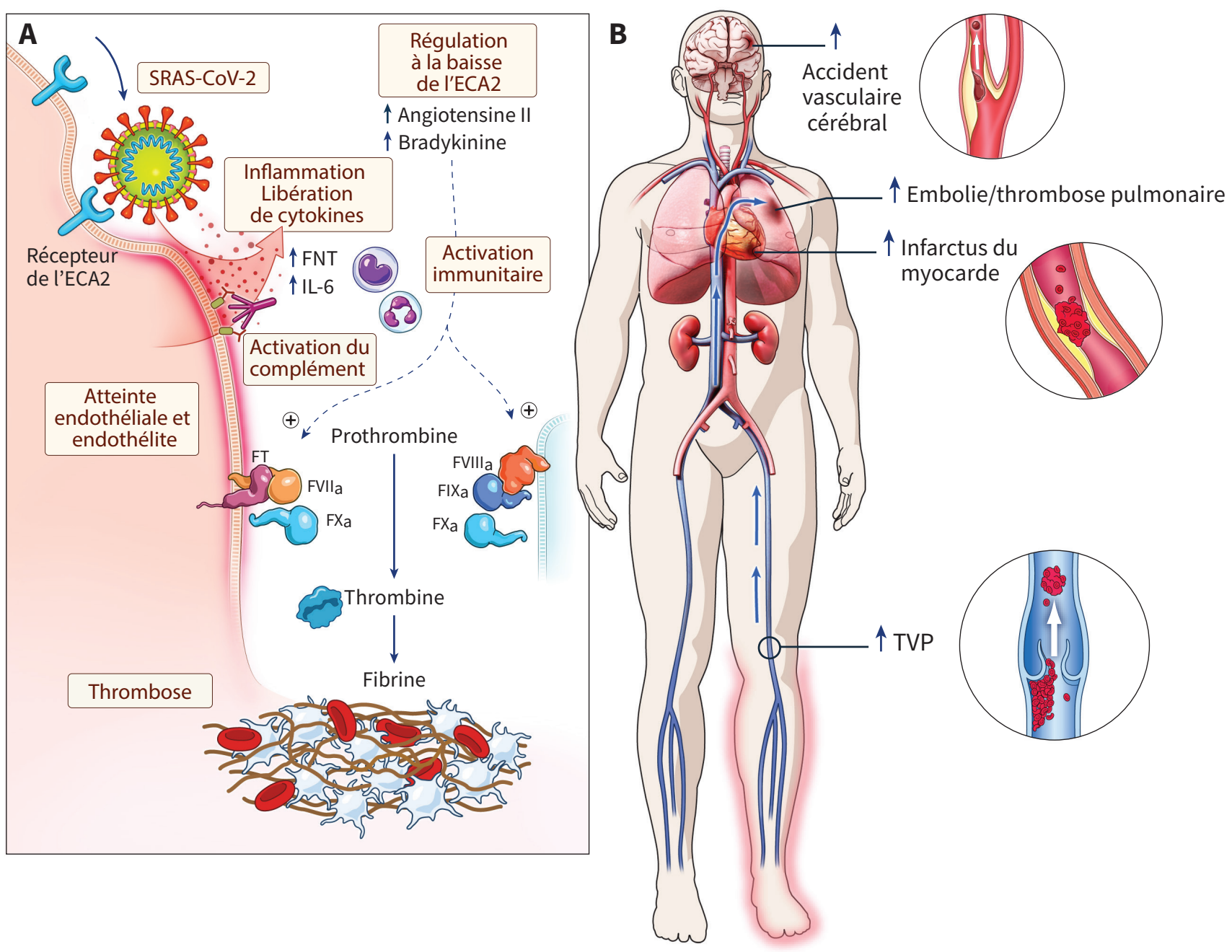

Figure 1 : Mécanismes possibles de la thrombose dans la maladie à coronavirus 2019 (COVID-19) et répercussions cliniques. (A) L'atteinte endothéliale déclenchée par l'entrée du coronavirus du syndrome respiratoire aigu sévère 2 (SRAS-CoV-2) dans les cellules par le biais du récepteur de l'enzyme de conversion de l'angiotensine 2 (ECA2) entraînerait une endothélite diffuse. L'atteinte endothéliale peut occasionner une réponse inflammatoire chez l'hôte, caractérisée par une activation immunitaire excessive et un orage cytokinique, qui est propice à l'hypercoagulabilité et à la thrombose. (B) Complications thrombotiques veineuses et artérielles possibles associées à la COVID-19. Remarque : EP = embolie pulmonaire, FNT = facteur de nécrose tissulaire $\alpha, \mathrm{FT}=$ facteur tissulaire, FVIIa = facteur VII activé, IL-6 = interleukine 6, TVP = thrombose veineuse profonde. Illustration originale exécutée par l'illustratrice médicale Gail Rudakevich. 


\section{Quelles sont les manifestations cliniques potentielles de la coagulopathie liée à la COVID-19?}

\section{Événements thrombotiques veineux}

Les événements thrombotiques veineux semblent fréquents chez les patients atteints de COVID-1924,25 (figure 1B). Dans une série de 107 cas consécutifs de COVID-19 admis dans une unité de soins intensifs (USI) en France, $20 \%$ ont présenté une thromboembolie veineuse (TEV), malgré une thromboprophylaxie pharmacologique standard (et une anticoagulothérapie à dose complète chez 2 patients) ${ }^{26}$. Comparativement à des témoins historiques (y compris des patients atteints d'insuffisance respiratoire d'origine grippale), les patients atteints de COVID-19 ont été au moins 2 fois plus susceptibles de présenter une TEV au cours d'une période de suivi équivalente ${ }^{26}$. Dans une étude de cohorte distincte, assortie selon le score de propension, en France, les patients atteints de COVID-19 souffrant d'un syndrome de détresse respiratoire aigu étaient 6 fois plus susceptibles de présenter une embolie pulmonaire comparativement aux patients dont le syndrome de détresse respiratoire aigu résultait d'autres cause ${ }^{23}$. Identifier les marqueurs du risque accru de thrombose et des complications thrombotiques qui peuvent s'ensuivre aiderait les médecins à prévoir ce type de complications. Des études ont avancé que certains biomarqueurs et la gravité de la maladie seraient des marqueurs du risque accru de thrombose. Par exemple, aux PaysBas, parmi 198 patients hospitalisés pour COVID-19, dont $17 \%$ ont reçu un diagnostic de TEV, l'augmentation du rapport neutrophiles:lymphocytes et des taux de D-dimères a été associée de manière indépendante à une incidence plus élevée de TEV, qui, en retour, a multiplié le risque de décès par un facteur de $2,4^{27}$. En revanche, les facteurs de risque cliniques, tels que l'obésité et les antécédents d'événements thrombotiques, n'ont pas été associés à une incidence accrue de TEV27. Aux États-Unis, une étude d'observation multicentrique sur 400 patients hospitalisés pour COVID-19 (dont 144 ont séjourné dans une USI) a constaté un taux de 4,8\% d'événements thrombotiques veineux confirmés à la radiographie et un taux de 9,5\% d'événements thrombotiques globaux durant le séjour hospitalier ${ }^{4}$. Dans cette série, des taux de D-dimères $>2500 \mathrm{ng} / \mathrm{mL}$, une numération plaquettaire $>450 \times 10^{9} / \mathrm{L}$, un taux de protéine $C$ réactive $>100 \mathrm{mg} / \mathrm{L}$ et une vitesse de sédimentation des érythrocytes $>40 \mathrm{~mm} / \mathrm{h}$ ont tous été associés de manière indépendante à une plus grande probabilité d'événements thrombotiques durant le séjour hospitalier ${ }^{4}$. Comme la plupart des études sur la prévalence des événements thrombotiques veineux liés à la COVID-19, cette étude est limitée du fait qu'elle était observationnelle et ne comportait pas de protocole universel ou systématique de dépistage de tels événements.

La fréquence des thromboses avérées est nettement plus élevée chez les patients atteints de COVID-19 que chez les patients souffrant d'autres maladies graves ${ }^{28}$. Comparativement à des patients atteints de pneumonie bactérienne extrahospitalière, les patients atteints de COVID-19 présentaient des taux de $D$-dimères plus élevés et des taux de protéine $C$ réactive plus bas au moment de leur hospitalisation ${ }^{29}$. Dans une série d'autopsies réalisées en Allemagne, des thromboses veineuses profondes bilatérales ont été observées chez 7 patients sur 12 atteints de COVID-19, et l'embolie pulmonaire massive a été la cause directe du décès de 4 d'entre eux ${ }^{30}$. La thrombose veineuse entraînant des anomalies régionales de la perfusion pulmonaire, associée à une dysfonction endothéliale et à une vasoconstriction pulmonaire hypoxémique, peut aussi expliquer l'hypoxie sévère observée malgré une compliance normale ou quasi-normale de l'appareil respiratoire chez certains patients ${ }^{31}$.

\section{Événements thrombotiques artériels}

La hausse du risque d'événements thrombotiques artériels serait aussi une caractéristique importante de la COVID-19. Dans une cohorte de 214 patients (âge moyen 53 ans, $41 \%$ de sexe masculin) atteints de COVID-19 à Wuhan, en Chine, la thrombose artérielle a frappé 6 patients ${ }^{32}$. La thrombose artérielle a également été signalée dans une série de cas, soit 5 patients âgés de 33 à 49 ans, à New York ${ }^{33}$. La thrombose in situ des petits vaisseaux, la thrombose embolique et les occlusions in situ des gros vaisseaux sont des mécanismes possibles de la thrombose chez les patients atteints de COVID-1934.

Une atteinte cardiaque (définie par un taux de troponine sérique de haute sensibilité au-delà du $99^{\text {e }}$ percentile de la limite supérieure de la normale) serait fréquente chez les patients atteints de COVID-19 et est associée à un pronostic nettement plus sombre ${ }^{35,36}$. Sur 416 patients hospitalisés pour COVID-19, environ $20 \%$ présentaient une lésion cardiaque ${ }^{35}$. La lésion cardiaque a été associée à un risque accru de complications, y compris d'insuffisance rénale aiguë, de coagulopathie et de décès (ce dernier étant 3,4 fois élevé chez les patients présentant une atteinte cardiaque) ${ }^{35}$. Les complications cardiaques de la COVID19 pourraient être liées à la forte expression de l'ECA2 dans les cellules cardiaques, y compris dans les cardiomyocytes, les fibroblastes et les cellules endothéliales coronariennes ${ }^{37}$, facilitant l'entrée du SRAS-CoV-2 dans ces types de cellules. L'atteinte cardiaque peut résulter d'une thrombose coronarienne micro- ou macrovasculaire (infarctus du myocarde), à une ischémie liée à la demande, à une cardiomyopathie de stress, à la myocardite et à une insuffisance cardiaque droite, entre autres causes ${ }^{38}$; de nombreuses autres étiologies peuvent être liées à l'hypercoagulabilité. L'hypercoagulabilité chez les patients atteints de COVID-19 jouerait aussi un rôle dans l'apparition des phénomènes aigus d'insuffisance rénale et d'ischémie mésentérique ${ }^{39,40}$ et périphérique qui ont été rapportés ${ }^{41,42}$.

\section{Thrombose microvasculaire}

Des cas de coagulation intravasculaire disséminée ont été signalés en lien avec la COVID-19 sévère. Dans une cohorte de 183 cas consécutifs d'hospitalisation pour COVID-19, 11,5\% $(n=21)$ sont décédés, 42,6\% ( $n=78)$ ont reçu leur congé et 45,9\% ( $n=84)$ étaient toujours à l'hôpital au dernier jour du suivi. En tout, $71 \%(n=15)$ des patients décédés répondaient aux critères diagnostiques de l'International Society on Thrombosis and Hemostasis pour la coagulation intravasculaire disséminée, contre 1 seul patient $(0,6 \%)$ parmi ceux qui ont reçu leur congé de l'hôpital ${ }^{2}$, mais la petite taille de l'échantillon et le fait que de nombreux patients 
étaient toujours à l'hôpital à la fin de la période de l'étude pourraient limiter l'extrapolation des résultats ${ }^{2}$. Les événements hémorragiques chez les patients atteints de COVID-19 semblent peu fréquents, mais le cas échéant, ils sont probablement liés à une coagulation intravasculaire disséminée ${ }^{43,44}$.

La coagulation intravasculaire peut être généralisée, mais parfois, elle est plus marquée ou grave au niveau des vaisseaux pulmonaires $^{6}$. Les taux hétérogènes de thrombose veineuse profonde observés à ce jour chez les patients atteints de COVID$19^{30,45,46}$ donnent à penser que certains événements thrombotiques pulmonaires seraient causés par une thrombose in situ plutôt que par une embolie, mais il faudra approfondir les études à ce sujet ${ }^{47}$. Comparativement aux patients décédés d'une pneumonie lors de la pandémie de grippe A H1N1 en 2009, les patients atteints de COVID-19 décédés d'insuffisance respiratoire étaient 9 fois plus nombreux à présenter des microthrombi au niveau des capillaires alvéolaires ${ }^{48}$. La protéase ADAMTS-13 (a disintegrin and metalloprotease with thrombospondin type motifs, member 13) est responsable du clivage des volumineux multimères hautement thrombogènes du facteur de Von Willebrand, et son absence peut entraîner le purpura thrombocytopénique thrombotique, une maladie prothrombotique ${ }^{49}$. Il reste à clarifier si la réduction de l'ADAMTS-13 constitue un médiateur important du dérèglement microcirculatoire et contribue à la dysfonction plurisystémique dans la COVID-19, comme cela a été démontré dans le choc septique ${ }^{50}$.

\section{Quels biomarqueurs peuvent faciliter le diagnostic de la coagulopathie et les traitements potentiels?}

Entre autres anomalies des analyses de laboratoire fréquemment associées à la coagulopathie liée à la COVID-19, mentionnons l'augmentation des taux de $D$-dimères et de fibrinogène ${ }^{7,10}$. Plus rarement, une baisse du fibrinogène et des numérations plaquettaires associée à un allongement du temps de prothrombine peut survenir et suggère un diagnostic de coagulation intravasculaire disséminée ${ }^{2}$. L'allongement du temps de thromboplastine partielle activée pourrait indiquer la présence d'un anticorps anticoagulant lupique ${ }^{22}$, lui-même associé à un état prothrombotique.

Le taux de D-dimères pourrait servir de marqueur d'un risque de thrombose chez les patients atteints de COVID-19, et ce marqueur a été proposé pour orienter les prises de décision clinique en ce qui concerne la thromboprophylaxie. Dans une étude sur 1099 patients atteints de COVID-19 à Wuhan, en Chine, $46 \%$ présentaient des taux de $D$-dimères $\geq$ à $0,5 \mathrm{mg} / \mathrm{L}^{51}$ au départ et chez 3169 patients de New York hospitalisés pour COVID-19, le taux médian de D-dimères à l'admission était élevé (438 ng/mL [éventail interquartile $262-872$; référence $<229$ ]) $)^{52}$. Suivant l'avis des experts et les données probantes mentionnées plus haut ${ }^{2}$, l'International Society on Thrombosis and Hemostasis recommande de mesurer les taux de D-dimères chez les patients hospitalisés pour COVID-19 afin d'aider à la stratification du risque ${ }^{53}$. Des taux élevés de D-dimères lors de l'hospitalisation ont été associés à une mortalité plus élevée ${ }^{54-56}$.

\section{Quelle est la meilleure stratégie antithrombotique pour les patients atteints de COVID-19?}

Conformément aux recommandations pour les soins aux patients hospitalisés en médecine ${ }^{57}$, les recommandations actuelles fondées sur des données probantes soulignent l'utilisation à grande échelle de l'anticoagulothérapie à dose thromboprophylactique chez les patients hospitalisés pour COVID-19, indépendamment de la gravité de la maladie ${ }^{53,58}$. Si on ne soupçonne pas de TEV clinique, on dispose de preuves insuffisantes pour recommander le dosage des $\mathrm{D}$-dimères afin de déterminer si les patients atteints de COVID19 ont besoin d'une anticoagulothérapie à haute dose ou non. Une étude d'observation rétrospective de Wuhan a laissé entendre que, comparativement à l'absence d'héparinothérapie, l'héparine (aux doses faibles couramment utilisées en thromboprophylaxie) était associée à une mortalité moindre à 28 jours chez les patients dont les taux de D-dimères étaient élevés ou qui présentaient un score de coagulation intravasculaire disséminée élevé ${ }^{59}$. Les auteurs de l'étude n'ont pas précisé la dose d'héparine utilisée (plus probablement, les patients du groupe sous héparine ont reçu des doses prophylactiques) et il semble que peu d'autres centres soient susceptibles de ne pas recourir à la thromboprophylaxie, comme dans le groupe de comparaison de cette étude ${ }^{59}$.

Des événements thrombotiques ont été signalés chez des patients atteints de COVID-19 recevant de l'héparine aux doses thromboprophylactiques standard ${ }^{3,23,26,27}$. Il y a donc lieu de se demander si des doses plus fortes d'héparine pourraient être bénéfiques chez ces patients. Dans une étude de cohorte rétrospective sur 2773 patients atteints de COVID-19 hospitalisés à New York, l'anticoagulothérapie (orale, sous-cutanée ou intraveineuse) a été associée à une meilleure survie ${ }^{60}$. La prolongation du traitement anticoagulant a été associée à une mortalité moindre chez les patients sous ventilation mécanique (risque relatif ajusté 0,86 par jour, IC à 95\% 0,82-0,89, $p<0,001$ ). Trois pour cent des sujets anticoagulés ont présenté un événement hémorragique, contre $2 \%$ chez ceux qui ne recevaient pas d'anticoagulants $(p=0,2)^{60}$. Toutefois, les limites méthodologiques de l'étude incluaient le biais de temps immortel et le biais d'indication qui limitent la capacité des médecins à tirer des inférences à partir des observations.

Outre ses effets anticoagulants, l'héparine peut également avoir d'autres effets bénéfiques, notamment la suppression des taux de cytokines inflammatoires, l'inhibition du complément et la suppression de la chimiotaxie et de la migration des neutrophiles ${ }^{61,62}$. L'héparine s'est déjà révélée capable de prévenir l'atteinte endothéliale en bloquant l'histone et l'ADN acellulaire et en protégeant l'étanchéité des jonctions endothéliales, ce qui diminue les fuites vasculaires et l'œdème pulmonaire durant la septicémie ${ }^{61}$. En présence de COVID-19, l'héparine a été associée à des taux moindres de $D$-dimères, de fibrinogène et d'interleukine 6 (une cytokine proinflammatoire) $)^{16,63}$. Selon une observation expérimentale intrigante, certaines héparines pourraient exercer des effets antiviraux directs : elles induiraient des changements de conformation de la protéine S du SRAS-CoV-2, ce qui l'empêcherait d'envahir les cellules et de se fixer à l'ECA2 ${ }^{64}$. Cet effet antiviral n'a cependant pas encore été démontré dans le cadre d'une étude clinique. 


\section{Quelles questions cliniques restent en suspens?}

L'observation de ces mécanismes et phénomènes cliniques a mené à plusieurs essais randomisés et contrôlés qui vérifient actuellement l'hypothèse selon laquelle l'héparine à dose thérapeutique améliorerait les résultats chez les patients atteints de COVID-19. L'étude ATTACC (Antithrombotic Therapy to Ameliorate Complications of COVID-19; NCT04372589) est un essai international émanant du Canada, qui recrute des patients hospitalisés pour la COVID-19 mais non gravement atteints, au Canada, aux États-Unis, au Brésil et au Mexique. L'étude procède à l'assignation aléatoire des patients à l'un de deux groupes, soit héparine thérapeutique pendant 14 jours ou soins usuels, et elle suit un protocole bayésien d'assignation aléatoire à réponse adaptée conçu pour répartir les sujets entre le groupe traité et le groupe témoin selon les données probantes qui s'accumulent sur la réponse au traitement en fonction des taux de D-dimères au départ. D'autres essais (NCT04362085, NCT04359277) évaluent l'effet de l'anticoagulothérapie chez les patients qui ont un taux de D-dimères élevé.

Deux essais internationaux portent sur l'impact de l'anticoagulothérapie chez les patients gravement malades qui ont besoin de ventilation artificielle, avec ou sans soutien pour défaillance plurisystémique. L'anticoagulothérapie spécifique à la COVID-19 sévère est évaluée dans la strate pandémique de l'essai international REMAP-CAP (Randomized, Embedded, Multifactorial Adaptive Platform trial for Community-Acquired Pneumonia; NCT02735707), et l'étude HALO (Heparin Anticoagulation in Septic Shock trial; NCT03378466) évalue l'héparine non fractionnée en dose thérapeutique chez des patients victimes de choc vasoplégique, dont certains sont atteints de COVID-19 sévère. Les résultats de ces essais permettront de vérifier si l'anticoagulothérapie héparinique est supérieure à l'héparine à dose standard chez des patients atteints de COVID-19 modérée ou sévère. Le cas échéant, le recours à l'application thérapeutique de l'héparine pourrait se répandre rapidement, y compris dans les pays à revenu faible et moyen étant donné que le médicament est relativement peu coûteux et facilement accessible.

L'utilité d'une anticoagulation à dose "intermédiaire ", l'impact des antiplaquettaires ${ }^{65}$ et l'avantage des schémas antithrombotiques après le congé sont aussi des questions importantes qui justifient la réalisation d'essais cliniques, dont certains sont prévus ou en cours. On a suggéré d'envisager la thromboprophylaxie après le congé hospitalier chez des patients atteints de COVID-19 exposés à un risque plus élevé d'événements thrombotiques veineux (p. ex., patients à mobilité réduite) ou chez ceux qui ont reçu une ventilation mécanique ${ }^{66}$. Par contre, il n'existe aucune donnée solide pour appuyer une recommandation à grande échelle, et par conséquent, une approche individualisée qui tient compte des risques est plus appropriée ${ }^{67,68}$. Les plaquettes jouent un rôle important dans les événements thromboemboliques, et les antiplaquettaires comme l'acide acétylsalicylique et le ticagrelor ${ }^{66,70}$ se sont révélés prometteurs pour ce qui est de réduire le risque d'événements thrombotiques chez les patients souffrant d'infection respiratoire. Très peu d'études ont exploré le rôle possible des antiplaquettaires chez les patients atteints de COVID- $19^{71}$ et pour l'instant, on ne peut pas formuler de recommandations officielles à ce sujet ${ }^{67}$.

\section{Conclusion}

La thrombose est une caractéristique importante chez les patients atteints de COVID-19. Étant donné les mécanismes antithrombotiques, anti-inflammatoires et peut-être antiviraux de l'héparine, l'anticoagulothérapie pourrait se révéler fort bénéfique, mais il n'a pas encore été démontré que ses bienfaits en surclassent les risques. D'autres ressources pour les médecins et les patients sont proposées dans l'encadré $n^{\circ} 2$. Nous attendons impatiemment les résultats des essais cliniques pour orienter le traitement optimal des patients atteints de COVID-19, peu importe leur degré d'atteinte.

Encadré $n^{\circ} 2$ : Ressources additionnelles pour les patients et les médecins

Directives cliniques (en anglais seulement)

- Clinical Guidance on the Diagnosis, Prevention and Treatment of Venous Thromboembolism in Hospitalized Patients with COVID-19 (International Society on Thrombosis and Hemostasis [ISTH] $)^{72}$

- COVID-19 Treatment Guidelines from the United States National Institutes of Health ${ }^{68}$

- European Society of Cardiology Guidance for the Diagnosis and Management of CV [Cardiovascular] Disease during the COVID-19 Pandemic ${ }^{58}$

- ISTH Interim Guidance on Recognition and Management of Coagulopathy in COVID-1953

- Thromboembolism and Anticoagulant Therapy During the COVID-19 Pandemic: Interim Clinical Guidance from the Anticoagulation Forum (US) ${ }^{66}$

Autres ressources en ligne pour les médecins (en anglais seulement)

- COVID-19 Clinical Resources from Thrombosis Canada: https:// thrombosiscanada.ca/covid-19/

- COVID-19 Resource Center of the American Society of Hematology: https://hematology.org/covid-19

- ISTH Academy website (COVID-19 outbreak menu): https:// academy.isth.org/

\section{Essais en cours}

- Nous dirigeons les lecteurs vers Bikdeli et ses collaborateurs ${ }^{67}$ pour une liste des essais cliniques en cours au sujet de la COVID19 et des traitements antithrombotiques. Nous leur suggérons également d'interroger la plateforme clinicaltrials.gov (https:// clinicaltrials.gov/ct2/results?cond = COVID-19), la base de données des études sur la COVID-19 de l'Organisation mondiale de la santé (https://clinicaltrials.gov/ct2/who_table), le Registre des essais cliniques européens (www.clinicaltrialsregister.eu/ ctr-search/search?query = covid-19) et The Global Coronavirus COVID-19 Clinical Trial Tracker (www.covid19-trials.com/)

\section{Ressources pour les patients}

- On peut trouver des ressources pour les patients sur le site Web North American Thrombose Forum, qui inclut des liens vers des ressources pour les patients offerts par plusieurs organisations: https://natfonline.org/covid-19-updates/patient-resources/ 


\section{Références}

1. Wu Z, McGoogan JM. Characteristics of and important lessons from the coronavirus disease 2019 (COVID-19) outbreak in China: summary of a report of 72314 cases from the Chinese Center for Disease Control and Prevention. JAMA 2020 Feb. 24 [Cyberpublication avant impression]. doi: 10.1001/ jama.2020.2648.

2. Tang N, Li D, Wang X, et al. Abnormal coagulation parameters are associated with poor prognosis in patients with novel coronavirus pneumonia. J Thromb Haemost 2020;18:844-7.

3. Klok FA, Kruip MJHA, van der Meer NJM, et al. Incidence of thrombotic complications in critically ill ICU patients with COVID-19. Thromb Res 2020;191:145-7.

4. Al-Samkari H, Karp Leaf RS, Dzik WH, et al. COVID and coagulation: bleeding and thrombotic manifestations of SARS-CoV2 Infection. Blood 2020;136: 489-500.

5. Varga Z, Flammer AJ, Steiger $P$, et al. Endothelial cell infection and endotheliitis in COVID-19. Lancet 2020;395:1417-8.

6. McGonagle D, O'Donnell JS, Sharif K, et al. Immune mechanisms of pulmonary intravascular coagulopathy in COVID-19 pneumonia. The Lancet Rheumatology 2020;2:E437-45. doi: 10.1016/S2665-9913(20)30121-1.

7. Levi M, Thachil J, Iba T, et al. Coagulation abnormalities and thrombosis in patients with COVID-19. Lancet Haematol 2020;7:e438-40.

8. Cao W, Li T. COVID-19: towards understanding of pathogenesis. Cell Res 2020;30: 367-9.

9. Teuwen L-A, Geldhof V, Pasut A, et al. COVID-19: the vasculature unleashed. Nat Rev Immunol 2020;20:389-91.

10. Connors JM, Levy JH. COVID-19 and its implications for thrombosis and anticoagulation. Blood 2020;135:2033-40.

11. Hoffmann M, Kleine-Weber H, Schroeder S, et al. SARS-CoV-2 cell entry depends on ACE2 and TMPRSS2 and is blocked by a clinically proven protease inhibitor. Cell 2020;181:271-80.e8.

12. Monteil V, Kwon H, Prado P, et al. Inhibition of SARS-CoV-2 infections in engineered human tissues using clinical-grade soluble human ACE2. Cell 2020;181: 905-13.e7.

13. Ahmed S, Anirvan P. Reply to rheumatologists' perspective on coronavirus disease 19: Is heparin the dark horse for COVID-19? Clin Rheumatol 2020;39: 2099-100.

14. Jackson SP, Darbousset R, Schoenwaelder SM. Thromboinflammation: challenges of therapeutically targeting coagulation and other host defense mechanisms. Blood 2019;133:906-18.

15. Maier CL, Truong AD, Auld SC, et al. COVID-19-associated hyperviscosity: a link between inflammation and thrombophilia? Lancet 2020;395:1758-9.

16. Ranucci M, Ballotta A, Di Dedda U, et al. The procoagulant pattern of patients with COVID-19 acute respiratory distress syndrome. J Thromb Haemost 2020;18: 1745-51.

17. Magro C, Mulvey JJ, Berlin D, et al. Complement associated microvascular injury and thrombosis in the pathogenesis of severe COVID-19 infection: a report of five cases. Transl Res 2020;220:1-13.

18. Campbell CM, Kahwash R. Will complement inhibition be the new target in treating COVID-19-related systemic thrombosis? Circulation 2020;141:1739-41.

19. Noris M, Benigni A, Remuzzi G. The case of complement activation in COVID-19 multiorgan impact. Kidney Int 2020;98:314-22.

20. Keragala CB, Draxler DF, McQuilten ZK, et al. Haemostasis and innate immunity - a complementary relationship. Br J Haematol 2018;180:782-98.

21. Zhang Y, Xiao M, Zhang S, et al. Coagulopathy and antiphospholipid antibodies in patients with COVID-19. N Engl J Med 2017;382:e38.

22. Bowles L, Platton S, Yartey N, et al. Lupus anticoagulant and abnormal coagulation tests in patients with COVID-19. N Engl J Med 2020;383:288-90.

23. Helms J, Tacquard C, Severac F, et al.; CRICS TRIGGERSEP Group. (Clinical Research in Intensive Care and Sepsis Trial Group for Global Evaluation and Research in Sepsis). High risk of thrombosis in patients with severe SARSCoV-2 infection: a multicenter prospective cohort study. Intensive Care Med 2020;46:1089-98.

24. Lodigiani C, lapichino G, Carenzo L, et al.; Humanitas COVID-19 Task Force. Venous and arterial thromboembolic complications in COVID-19 patients admitted to an academic hospital in Milan, Italy. Thromb Res 2020;191:9-14.

25. Lax SF, Skok K, Zechner P, et al. Pulmonary arterial thrombosis in COVID-19 with fatal outcome: results from a prospective, single-center, clinicopathologic case series. Ann Intern Med 2020 May 14 [Cyberpublication avant impression]. doi: $10.7326 /$ M20-2566
26. Poissy J, Goutay J, Caplan M, et al.; Lille ICU Haemostasis COVID-19 Group. Pulmonary embolism in COVID-19 patients: awareness of an increased prevalence. Circulation 2020;142:184-6.

27. Middeldorp S, Coppens M, van Haaps TF, et al. Incidence of venous thromboembolism in hospitalized patients with COVID-19. J Thromb Haemost 2020 May 5 [Cyberpublication avant impression]. doi: 10.1111/jth.14888.

28. The PROTECT Investigators for the Canadian Critical Care Trials Group and the Australian and New Zealand Intensive Care Society Clinical Trials Group; Cook D, Meade M, Guyatt G, et al. Dalteparin versus unfractionated heparin in critically ill patients. N Engl J Med 2011;364:1305-14.

29. Yu B, Li X, Chen J, et al. Evaluation of variation in D-dimer levels among COVID-19 and bacterial pneumonia: a retrospective analysis. J Thromb Thrombolysis 2020 June 10 [Cyberpublication avant impression]. doi: 10.1007/s11239-020-02171-y.

30. Wichmann D, Sperhake J-P, Lutgehetmann M, et al. Autopsy findings and venous thromboembolism in patients with COVID-19. Ann Intern Med 2020 May 6 [Cyberpublication avant impression]. doi: 10.7326/M20-2003.

31. Leisman DE, Deutschman CS, Legrand M. Facing COVID-19 in the ICU: vascular dysfunction, thrombosis, and dysregulated inflammation. Intensive Care Med 2020;46:1105-8.

32. Mao L, Jin H, Wang M, et al. Neurologic manifestations of hospitalized patients with coronavirus disease 2019 in Wuhan, China. JAMA Neurol 2020 April 10. [Cyberpublication avant impression]. doi: 10.1001/jamaneurol.2020.1127.

33. Oxley TJ, Mocco J, Majidi S, et al. Large-vessel stroke as a presenting feature of COVID-19 in the young. N Engl J Med 2020;382:e60.

34. Berekashvili K, Dmytriw AA, Vulkanov V, et al. Etiologic subtypes of ischemic stroke in SARS-COV-2 virus patients. medRxiv 2020 May 15. doi: 10.1101/2020.05.03 .20077206.

35. Shi S, Qin M, Shen B, et al. Association of cardiac injury with mortality in hospitalized patients with COVID-19 in Wuhan, China. JAMA Cardiol 2020;5:802-10.

36. Shi S, Qin M, Cai Y, et al. Characteristics and clinical significance of myocardial injury in patients with severe coronavirus disease 2019. Eur Heart J 2020;41: 2070-9.

37. Guo J, Huang Z, Lin L, et al. Coronavirus disease 2019 (COVID-19) and cardiovascular disease: a viewpoint on the potential influence of angiotensin-converting enzyme inhibitors/angiotensin receptor blockers on onset and severity of severe acute respiratory syndrome coronavirus 2 infection. J Am Heart Assoc 2020;9:e016219.

38. Bikdeli B, Madhavan MV, Jimenez D, et al.; Global COVID-19 Thrombosis Collaborative Group, Endorsed by the ISTH, NATF, ESVM, and the IUA, Supported by the ESC Working Group on Pulmonary Circulation and Right Ventricular Function. COVID-19 and thrombotic or thromboembolic disease: implications for prevention, antithrombotic therapy, and follow-u,p: JACC state-of-the-art review. J Am Coll Cardiol 2020;75:2950-73.

39. Batlle D, Soler MJ, Sparks MA, et al.; COVID-19 and ACE2 in Cardiovascular, Lung, and Kidney Working Group. Acute kidney injury in COVID-19: emerging evidence of a distinct pathophysiology. J Am Soc Nephrol 2020;31:1380-3.

40. Lushina N, Kuo JS, Shaikh HA. Pulmonary, cerebral, and renal thromboembolic disease associated with COVID-19 infection. JAMA 2020 April 23. [Cyberpublication avant impression] doi: 10.1148/radiol.2020201623.

41. Bellosta R, Luzzani L, Natalini G, et al. Acute limb ischemia in patients with COVID-19 pneumonia. J Am Coll Surg 2020 April 29. S0741-5214(20)31080-6. [Cyberpublication avant impression]. doi: 10.1016/j.jvs.2020.04.483.

42. Bhayana R, Som A, Li MD, et al. Abdominal imaging findings in COVID-19: preliminary observations. JAMA 2020 May 11. [Cyberpublication avant impression]. doi: 10.1148/radiol.2020201908.

43. Poyiadji N, Shahin G, Noujaim D, et al. COVID-19-associated acute hemorrhagic necrotizing encephalopathy: CT and MRI features. Radiology 2020;296:E119-20.

44. Lee SG, Fralick M, Sholzberg M. Coagulopathy associated with COVID-19. JAMC 2020;192:E583.

45. Nahum J, Morichau-Beauchant T, Daviaud F, et al. Venous thrombosis among critically ill patients with coronavirus disease 2019 (COVID-19). JAMA Netw Open 2020;3:e2010478.

46. Voicu S, Bonnin P, Stépanian A, et al. High prevalence of deep vein thrombosis in mechanically ventilated COVID-19 patients. J Am Coll Cardiol 2020;76:480-2.

47. Cattaneo M, Bertinato EM, Birocchi S, et al. Pulmonary embolism or pulmonary thrombosis in COVID-19? Is the recommendation to use high-dose heparin for thromboprophylaxis hustified? J Thromb Haemost 2020 April 29. [Cyberpublication avant impression]. doi: 10.1055/s-0040-1712097.

48. Ackermann M, Verleden SE, Kuehnel M, et al. Pulmonary vascular endothelialitis, thrombosis, and angiogenesis in COVID-19. N Engl J Med 2020;383:120-8. 
49. Morici N, Bottiroli M, Fumagalli R, et al. Role of von Willebrand factor and ADAMTS-13 in the pathogenesis of thrombi in SARS-CoV-2 infection: time to rethink. J Thromb Haemost 2020 June 23. [Cyberpublication avant impression]. doi: $10.1055 / \mathrm{s}-0040-1713400$

50. Levi M, Scully M, Singer M. The role of ADAMTS-13 in the coagulopathy of sepsis. J Thromb Haemost 2018;16:646-51.

51. Guan W-j, Ni Z-y, Hu Y, et al. Clinical Characteristics of Coronavirus Disease 2019 in China. N Engl J Med 2020;382:1708-20.

52. Richardson S, Hirsch JS, Narasimhan M, et al. Presenting characteristics, comorbidities, and outcomes among 5700 patients hospitalized with COVID-19 in the New York City area. JAMA 2020;323:2052-9.

53. Thachil J, Tang N, Gando S, et al. ISTH interim guidance on recognition and management of coagulopathy in COVID-19. J Thromb Haemost 2020;18:1023-6.

54. Zhou F, Yu T, Du R, et al. Clinical course and risk factors for mortality of adult inpatients with COVID-19 in Wuhan, China: a retrospective cohort study. Lancet 2020;395:1054-62.

55. Zhang L, Yan X, Fan Q, et al. D-dimer levels on admission to predict in-hospital mortality in patients with COVID-19. J Thromb Haemost 2020;18:1324-9.

56. Cummings MJ, Baldwin MR, Abrams D, et al. Epidemiology, clinical course, and outcomes of critically ill adults with COVID-19 in New York City: a prospective cohort study. Lancet 2020;395:1763-70.

57. Schünemann HJ, Cushman M, Burnett AE, et al. American Society of Hematology 2018 guidelines for management of venous thromboembolism: prophylaxis for hospitalized and nonhospitalized medical patients. Blood Adv 2018;2:3198-225.

58. Bhatt DL. CABG the clear choice for patients with diabetes and multivessel disease. Lancet 2018;391:913-4.

59. Tang N, Bai H, Chen X, et al. Anticoagulant treatment is associated with decreased mortality in severe coronavirus disease 2019 patients with coagulopathy. J Thromb Haemost 2020;18:1094-9.

60. Paranjpe I, Fuster V, Lala A, et al. Association of treatment dose anticoagulation with in-hospital survival among hospitalized patients with COVID-19. J Am Coll Cardiol 2020;76:122-4.

61. Thachil J. The versatile heparin in COVID-19. J Thromb Haemost 2020;18: 1020-2.
62. Liu J, Li J, Arnold K, et al. Using heparin molecules to manage COVID-2019. Res Pract Thromb Haemost 2020;4:518-23.

63. Shi $\mathrm{C}$, Wang $\mathrm{C}$, Wang $\mathrm{H}$, et al. The potential of low molecular weight heparin to mitigate cytokine storm in severe COVID-19 patients: a retrospective clinical study. medRxiv 2020 April 15. doi: 10.1101/2020.03.28.20046144.

64. Mycroft-West C, Su D, Elli S, et al. The 2019 coronavirus (SARS-CoV-2) surface protein (Spike) S1 Receptor Binding Domain undergoes conformational change upon heparin binding. bioRxiv 2020 March 2. doi: 10.1101/2020.02.29.971093.

65. Zhou X, Li Y, Yang Q. Antiplatelet therapy after percutaneous coronary intervention in patients with COVID-19: implications from clinical features to pathologic findings. Circulation 2020;141:1736-8.

66. Barnes GD, Burnett A, Allen A, et al. Thromboembolism and anticoagulant therapy during the COVID-19 pandemic: interim clinical guidance from the anticoagulation forum. J Thromb Thrombolysis 2020;50:72-81.

67. Bikdeli B, Madhavan MV, Gupta A, et al.; Global COVID-19 Thrombosis Collaborative Group. Pharmacological agents targeting thromboinflammation in COVID-19: review and implications for future research. Thromb Haemost 2020;120:1004-24.

68. Dilsizian V, Arrighi JA, Cohen RS, et al. COCATS 4 Task Force 6: training in nuclear cardiology. J Am Coll Cardiol 2015;65:1800-9.

69. Boyle AJ, Di Gangi S, Hamid UI, et al. Aspirin therapy in patients with acute respiratory distress syndrome (ARDS) is associated with reduced intensive care unit mortality: a prospective analysis. Crit Care 2015;19:109.

70. Sexton TR, Zhang G, Macaulay TE, et al. Ticagrelor reduces thromboinflammatory markers in patients with pneumonia. JACC Basic Trans/ Sci 2018;3:435-49.

71. Liu X, Li Z, Liu S, et al. Potential therapeutic effects of dipyridamole in the severely ill patients with COVID-19. Acta Pharm Sin B 2020 April 20. [Cyberpublication avant impression] doi: 10.1016/j.apsb.2020.04.008.

72. Spyropoulos AC, Levy JH, Ageno W, et al.; Subcommittee on Perioperative, Critical Care Thrombosis, Haemostasis of the Scientific, Standardization Committee of the International Society on Thrombosis, Haemostasis. Scientific and standardization committee communication: clinical guidance on the diagnosis, prevention and treatment of venous thromboembolism in hospitalized patients with COVID-19. J Thromb Haemost 2020 May 27. [Cyberpublication avant impression]. doi: $10.1111 /$ jth.14929.
Intérêts concurrents : Aucun intérêt concurrent déclaré

Cet article a été révisé par des pairs.

Affiliations : Centre de cardiologie Peter Munk (Godoy, Lawler), Université de Toronto, Toronto, Ont.; Instituto do Coracao (Godoy), Hospital das Clinicas HCFMUSP, Faculdade de Medicina, Universidade de Sao Paulo, Sao Paulo, Brésil; Division interdépartmentale de médecine de soins intensifs (Goligher, Lawler, Slutsky), Université de Toronto; Division de pneumologie (Goligher), Département de médecine, Réseau universitaire de santé; Institut de recherche de l'Hôpital général de Toronto (Goligher, Lawler); Centre de recherche Keenan (Slutsky), Institut du savoir Li Ka Shing,
Hôpital St. Michael's, Toronto, Ont.; Faculté des sciences de la santé Max Rady, Faculté de médecine Max Rady (Zarychanski), Département de médecine interne, Université du Manitoba; Institut de recherche en oncohématologie (Zarychanski), CancerCare Manitoba, Winnipeg, Man.

Collaborateurs : Tous les auteurs ont contribué à l'élaboration et à la conception des travaux. Lucas Godoy, Ewan Goligher et Patrick Lawler ont contribué à la collecte, à l'analyse et à l'interprétation des données. Tous les auteurs ont participé à la rédaction du manuscrit, en ont révisé de façon critique le contenu intellectuel important, ont donné leur approbation finale pour la version destinée à être publiée, et assu- ment l'entière responsabilité de tous les aspects du travail. Arthur Slutsky et Ryan Zarychanski y ont contribué à parts égales.

Financement : Tous les auteurs sont des investigateurs de l'essai ATTACC (NCT04372589), qui est subventionné par les Instituts de recherche en santé du Canada (IRSC), Research Manitoba, le Centre de cardiologie Peter Munk et la Fondation Thistledown. Ewan Goligher bénéficie de l'appui d'une subvention pour chercheur en début de carrière des IRSC (AR7-162822). Arthur Slutsky est détenteur de la chaire de médecine Keenan. Ryan Zarychanski est détenteur de la chaire Lyonel G. Israels, Université du Manitoba.

Correspondance : Patrick Lawler, patrick.lawler@uhn.ca 